\title{
Gastric Bezoar After Vagotomy and Billroth I Resection Presenting With Gastric Outlet Obstruction
}

\author{
Christine Boumitri ${ }^{\mathrm{a}}$, Liliane Deeb ${ }^{\mathrm{a}, \mathrm{b}}$
}

\begin{abstract}
Bezoars are collections of undigested materials that can accumulate in any part of the gastrointestinal (GI) tract with the stomach being the most frequent location. They could occur as a complication of gastric surgery, which creates a low acid environment, decreased peristalsis, and abnormal pyloric function. Vagotomy and Billroth resections are culprit surgeries even decades after the procedure. The clinical presentation varies from asymptomatic, a vague feeling of epigastric discomfort and bloating to gastric ulcerations from pressure necrosis and subsequent GI bleeding, or gastric outlet obstruction. Endoscopy is not only the diagnostic test of choice but also has the advantage of offering therapeutic interventions like fragmentation or dissolution. When conservative treatments and endoscopic approaches fail, surgical extraction is recommended. We describe a patient with a gastric bezoar 25 years after vagotomy and Billroth $\mathrm{I}$ in which the diagnosis was delayed due to atypical presentation.
\end{abstract}

Keywords: Gastric bezoar; Gastric outlet obstruction; Billroth I and vagotomy; Endoscopy; Surgery

\section{Introduction}

Bezoars are collections of undigested materials that can accumulate in any part of the gastrointestinal (GI) tract with the stomach being the most frequent location. Bezoars are named according to the primary constituent of the complex. Phytobezoars are formed of indigestible fibers such as cellulose, hemicellulose and lignin, which are common in celery, prune, pumpkin, and persimmon. Trichobezoars consist of hair collections that are common in women and psychiatric patients. Pharmacobezoars are composed of medications or vitamins de-

Manuscript accepted for publication September 17, 2015

aDepartment of Gastroenterology, Staten Island University Hospital, Staten Island, NY, USA

${ }^{\mathrm{b}}$ Corresponding Author: Liliane Deeb, Staten Island University Hospital, 475 Seaview Avenue, Staten Island, NY 10305, USA.

Email: 1dkhouri@hotmail.com

doi: http://dx.doi.org/10.14740/jmc2310w signed to resist digestion. Lastly lactobezoars are made of undigested milk and are mostly described in infants and toddlers.

Classically, most bezoars occur as a complication of gastric surgery, which creates a low acid environment, decreased peristalsis, and abnormal pyloric function. The incidence of bezoars in adult patients has increased as a result of operative manipulation of the GI tract especially gastric bypass surgeries for bariatric purposes. Billroth I resections that have become a historical operation to treat peptic ulcers with the advent of proton pump inhibitors, have a high incidence of gastric bezoars, especially if accompanied by vagotomy. Because of the change in GI anatomy, these patients may not present with typical signs and symptoms of bowel obstruction. Delay in diagnosis may lead to futile complications. We report a case of gastric bezoar in a patient with history of Billroth I surgery and vagotomy presenting with gastric outlet obstruction (GOO) that was mistaken at first for biliary colic.

\section{Case Report}

A 55-year-old man with history of peptic ulcer disease who underwent vagotomy and Billroth I gastric resection 25 years ago presented to our emergency department with progressively worsening intermittent postprandial right upper quadrant (RUQ) pain associated with nausea and vomiting of 9 months duration. He denied fever, chills, jaundice, weight loss and blood in stools. Initial physical exam revealed an afebrile patient with a blood pressure of $133 / 68 \mathrm{~mm} \mathrm{Hg}$, and a heart rate of 81. Abdominal examination revealed soft abdomen, tender to palpation in the RUQ without evidence of positive Murphy's sign, rebound tenderness or guarding. Laboratory tests showed normal transaminases, alkaline phosphatase, and mildly elevated total bilirubin of $1.4 \mathrm{mg} / \mathrm{dL}$ and low hemoglobin of $12.6 \mathrm{~g} / \mathrm{dL}$. All other tests (cell count, renal function, serum electrolytes and lactic acid) were unremarkable. At this point, the main differential was to rule out biliary disease. Abdominal ultrasound revealed gallbladder sludge without evidence of cholecystitis, a borderline common bile duct size measuring $7 \mathrm{~mm}$ and fatty infiltration of liver. A flat abdominal X-ray revealed non-obstructive gas pattern. Hepatobiliary iminodiacetic acid (HIDA) scan demonstrated definite visualization of the gallbladder. CT of abdomen and MRCP were unremarkable except for the upper limit size of the common bile duct at 


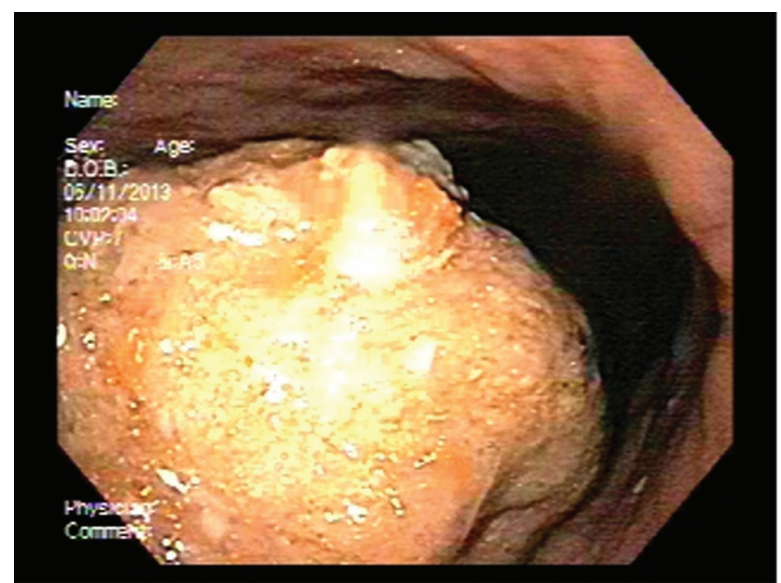

Figure 1. Large gastric bezoar identified during endoscopy.

$7 \mathrm{~mm}$. Patient was managed by bowel rest, IV hydration and antiemetics. Few days later, he tolerated oral intake and was discharged home for watchful waiting.

Three months later, abdominal pain and vomiting recurred. Revisiting the history at this point disclosed symptoms of GOO with vomiting of undigested food within an hour after food intake. Esophagogastroduodenoscopy was done and revealed a large bezoar in the gastric fundus (Fig. 1). Attempts to endoscopic fragmentation of the bezoar failed due to its hard consistency (Fig. 2). Patient was referred for laparoscopic surgical treatment. A $10 \times 10 \times 3 \mathrm{~cm}$ sized bezoar was successfully retrieved through a gastrostomy, using an endobag (Fig. 3). Pathology revealed a bundle of hair shafts mixed with food residue. Patient did well thereafter and symptoms completely resolved.

\section{Discussion}

Bezoars, from the Persian $p \bar{a} d-z a h r$, which means antidote, are stones that were thought to have a magical cure power against

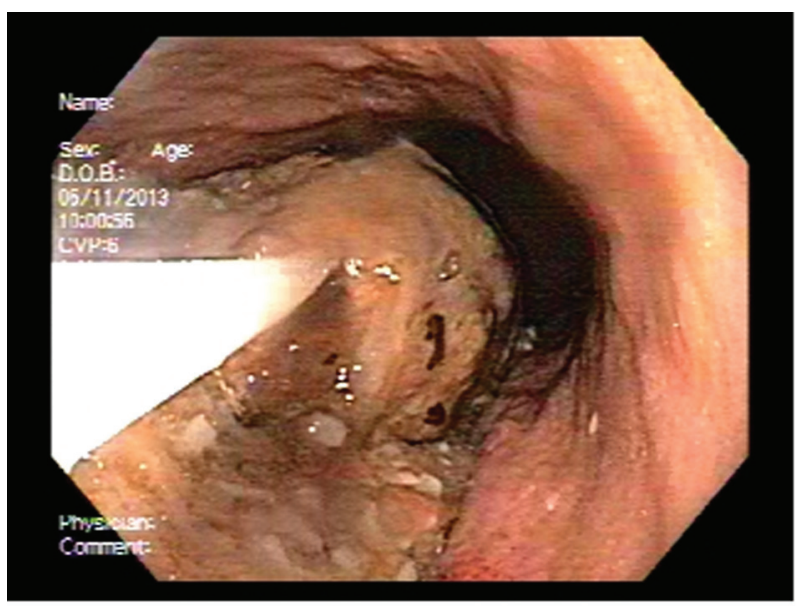

Figure 2. Multiple endoscopic attempts to fragment the bezoar with different tools.

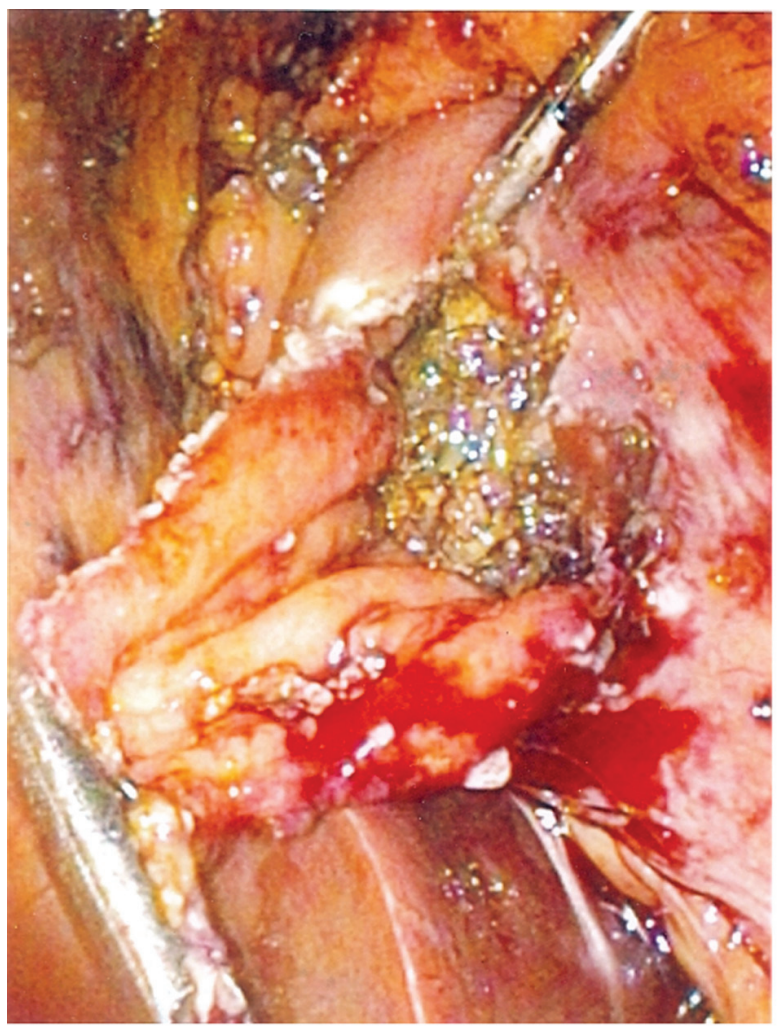

Figure 3. A gastrostomy showing retrieval of a $10 \times 10 \times 3 \mathrm{~cm}$ sized bezoar using an endobag.

plague, snake-bite, leprosy, and epilepsy in the old centuries. They were first described as such in 1161 by Ibn Zuhr, an Andalusian physician, till 1575 when Ambroise Pare, a general surgeon proved that the ability of these stones to cure diseases was a false claim.

A bezoar is also known as an enterolith, a concretion of foreign or indigestible matter found in the alimentary canal. As previously mentioned, they are classified according to the materials they are composed of, into phytobezoars, trichobezoars, and pharmacobezoars $[1,2]$. The components of two bezoars can rarely coexist to cause a phytotrichobezoar as in our patient. Foreign bodies bezoars (such as metal and plastic) and trichobezoars can occur in patient with normal gastric motility and with no predisposing medical conditions; however, the vast majority of bezoars occur in patients with previous surgeries or medical conditions that might affect the gastric emptying. Culprit surgeries include vagotomies and pyloroplasties which result in decreased acid secretion, delayed gastric emptying and loss of pyloric function [3]. Medical conditions that may predispose to bezoar formation are diabetic neuropathy, end-stage renal disease, myotonic dystrophy, Crohn's disease, gastric, duodenal or pancreatic carcinoma, peptic ulcer disease, strictures and hypothyroidism $[3,4]$.

Bezoars are a rare cause of GI obstruction and account for $0.4-4 \%$ of cases of small bowel obstruction [5]. Our patient had an unusual presentation of symptoms and signs of GOO secondary to a complex phytotrichobezoar. Patients with gastric bezoars have a clinical presentation ranging from asymp- 
tomatic to vague symptoms of epigastric discomfort, bloating, and anorexia to more serious presentations such as GOO, GI bleeding, intestinal obstruction and perforation [6, 7]. Rarely trichobezoars could present as Rapunzel syndrome where the bezoar is primarily located in the stomach and extends down past the pylorus to the duodenum to cause complete obstruction.

History remains a helpful tool in the diagnosis of bezoar. A history of psychiatric disorders, previous gastric surgeries, the amount and types of food or medications consumed might assist in the diagnosis. Clinical presentation is often non-specific and may mimic other pathological GI conditions.

Early diagnosis and aggressive treatment is the key to successful management of the condition, which is curable. EGD is the diagnostic test of choice for the diagnosis of esophageal, gastric or duodenal bezoars as in our patient. Other helpful diagnostic tools include computed tomography or flat X-ray that could show radiopaque bezoars.

Treatment depends on the location, size and composition of the bezoar. Small bezoars can be treated conservatively with liquid diet and prokinetic agents. Chemical dissolution by enteral administration of proteolytic enzymes or cola may as well be successful in small phytobezoars [8]. The administration of $\mathrm{N}$-acetylcysteine through nasogastric tube in infants and toddlers with gastric lactobezoars has been reported as successful, safe and non-invasive treatment of lactobezoars [9, 10]. When conservative management and chemical dissolution fail, the next step is typically endoscopic fragmentation. Forceps and snares with and without saline and water instillation have been used to break the bezoar. Fragments can be either pushed into the small bowel or removed if airway protection is available to prevent aspiration. In 1988, Yuang et al introduced the Nd:YAG laser-ignited mini-explosive technique to treat gastric bezoars with a non-invasive approach [11]. The success rate of this approach was reported to be as high as $99.6 \%$ with $84.9 \%$ cure rate after one treatment and $15.1 \%$ after two treatments with no significant intraoperative or delayed complications [12]. When endoscopic approach fails to resolve the bezoar or when GI obstruction occurs, surgical treatment is recommended with thorough exploration of the intestinal tract to avoid missing any retained bezoar [13]. Recently, the laparoscopic approach to removing the bezoar like what our patient had, through an anterior wall gastrostomy in an endobag, has become increasingly popular and slowly growing as the choice intervention [14].

\section{Conclusion}

Bezoar is a curable condition but can potentially cause significant morbidity and mortality. GOO secondary to bezoars is uncommon and remains a diagnostic challenge as in our case. The presentation can be atypical and the symptoms can be vague and may mimic other conditions. Bezoars should be suspected in patients with an increased risk, such as those with previous gastric surgery, gastric motility disorders, poor dentition, mental retardation and a suggestive history of increased fiber intake. Early diagnosis is warranted to reduce unneces- sary delays before appropriate management. Endoscopy is the choice modality of diagnosis, and endoscopic removal is an effective treatment modality; however, conventional surgical intervention is needed when endoscopic fragmentation fails. All clinicians should keep bezoar always in their differential especially when the history suggests the presence of predisposing factors.

\section{References}

1. Hall JD, Shami VM. Rapunzel's syndrome: gastric bezoars and endoscopic management. Gastrointest Endosc Clin N Am. 2006;16(1):111-119.

2. Kumar GS, Amar V, Ramesh B, Abbey RK. Bizarre metal bezoar: a case report. Indian J Surg. 2013;75(Suppl $1): 356-358$.

3. LaFountain J. Could your patient's bowel obstruction be a bezoar? Todays Surg Nurse. 1999;21(2):34-37.

4. Robles R, Parrilla P, Escamilla C, Lujan JA, Torralba JA, Liron R, Moreno A. Gastrointestinal bezoars. Br J Surg. 1994;81(7):1000-1001.

5. Ho TW, Koh DC. Small-bowel obstruction secondary to bezoar impaction: a diagnostic dilemma. World J Surg. 2007;31(5):1072-1078; discussion 1079-1080.

6. Goldstein SS, Lewis JH, Rothstein R. Intestinal obstruction due to bezoars. Am J Gastroenterol. 1984;79(4):313318.

7. Andrus $\mathrm{CH}$, Ponsky JL. Bezoars: classification, pathophysiology, and treatment. Am J Gastroenterol. 1988;83(5):476-478.

8. Gupta R, Share M, Pineau BC. Dissolution of an esophageal bezoar with pancreatic enzyme extract. Gastrointest Endosc. 2001;54(1):96-99.

9. Sparks B, Kesavan A. Treatment of a gastric lactobezoar with N-acetylcysteine. Case Rep Gastrointest Med. 2014;2014:254741.

10. Heinz-Erian P, Klein-Franke A, Gassner I, Kropshofer G, Salvador C, Meister B, Muller T, et al. Disintegration of large gastric lactobezoars by $\mathrm{N}$-acetylcysteine. J Pediatr Gastroenterol Nutr. 2010;50(1):108-110.

11. Huang YC, Guo ZH, Gu Y, Yang JQ, Liu QC, Cheng GY, Chen HL. Endoscopic lithotripsy of gastric bezoars using a laser-ignited mini-explosive device. Chin Med J (Engl). 1990;103(2):152-155.

12. Mao Y, Qiu H, Liu Q, Lu Z, Fan K, Huang Y, Yang Y. Endoscopic lithotripsy for gastric bezoars by $\mathrm{Nd}$ :YAG laser-ignited mini-explosive technique. Lasers Med Sci. 2014;29(3):1237-1240.

13. Robles Campos R, Parrilla Paricio P, Aguayo Albasini J, Riquelme Riquelme J, Cifuentes Tebar J, Lujan Mompean J, et al. Gastrointestinal bezoars: presentation of 60 cases. Digestive surgery. 1990;7(1):39-44.

14. Yau KK, Siu WT, Law BK, Cheung HY, Ha JP, Li MK. Laparoscopic approach compared with conventional open approach for bezoar-induced small-bowel obstruction. Arch Surg. 2005;140(10):972-975. 